\title{
Serum containing Buyang Huanwu decoction prevents age- associated migration and invasion of human vascular smooth muscle cells by up regulating SIRT1 expression
}

\author{
Li Zhang ${ }^{1, \S, *}$, Chunshan Wei ${ }^{2,}$, Yunjun Ruan ${ }^{3}$, Yanan Zhang $^{4}$, Yuliang Zhou ${ }^{1}$, Da Lei ${ }^{1, *}$ \\ ${ }^{1}$ Department of Cardiology, The First Affiliated Hospital of Guangdong Pharmaceutical University, Guangzhou, China; \\ ${ }^{2}$ Department of Liver Disease, Shenzhen Hospital Affiliated to Guangzhou University of Chinese Medicine, Shenzhen, China; \\ ${ }^{3}$ Department of Cardiology, Guangzhou General Hospital of Guangzhou Military Command, Guangzhou, China; \\ ${ }^{4}$ Veterinary medicine, Northeast Agricultural University, Haerbin, China.
}

\begin{abstract}
Summary The migration and invasion of vascular smooth muscle cells (VSMCs) caused by advanced aging play an important role in diffuse intimal thickening, facilitate adverse arterial remodeling and contribute to the initiation and progression of cardiovascular diseases. The inhibitory function of Buyang Huanwu decoction (BYHWD) has been found on aortic intimal hyperplasia and VSMC proliferation, but its effect on age-associated migration and invasion remains unknown. Here, we used an in vitro angiotensin II (Ang II)-induced senescence model to study the effects of serum containing BYHWD (BYHWS) on the migratory and invasive capacities, matrix metalloprotease type 2 (MMP-2) expression and modulation of sirtuin1 (SIRT1) signaling in human aorta VSMCs (HA-VAMCs). Our results showed that BYHWS was able to inhibit Ang II-induced migration and invasion, with down-regulation of MMP-2. In addition, manipulation of SIRT1 by either over-expression or siRNA knockdown ameliorated or promoted cellular migration and invasion, respectively. Moreover, BYHWS reversed senescence-mediated decrease of SIRT1 levels and SIRT1 was required for BYHWS regulation on migration and invasion of senescent HA-VAMCs. In summary, our data demonstrated that BYHWS suppressed the migration and invasion of age-associated VSMC via an increase of the SIRT1 level, which provides novel insights for the therapy of ageassociated cardiovascular diseases.
\end{abstract}

Keywords: Buyang Huanwu decoction, vascular smooth muscle cells, senescence, migration/ invasion, matrix metalloprotease type 2, sirtuin1 (SIRT1)

\section{Introduction}

Vascular aging has been viewed as a specific risk factor of cardiovascular diseases (CVD), such as atherosclerosis and hypertension (1-4). A complex series of events are involved in remodeled arterial wall with advanced aging, including migration and invasion of

Released online in J-STAGE as advance publication June 28, 2018.

${ }^{\S}$ These authors contributed equally to this work.

*Address correspondence to:

Dr Li Zhang and Da Lei, Department of Cardiology, The First Affiliated Hospital of Guangdong Pharmaceutical University, No. 19 Nonglinxia Road, Yuexiu District, Guangzhou, Guangdong 510080, China.

E-mail: zhangli4029@126.com and gyleida@163.com abnormal vascular smooth muscle cells (VSMC), which significantly contribute to diffuse intimal thickening and the onset and progression of CVD $(2,3)$. Previous data on interventions in age-associated VSMC migration and invasion indicated modulation of decrease and delay of the occurrence of severe CVD (4). New classes of drugs are currently being tested for CVD prevention, including glitazones and rimonabant. However, adverse effects such as heart failure and depression were found during the application of these treatments (5). Therefore, novel therapy remains to be developed.

It has been implicated that traditional Chinese Medicine (TCM) exerts a regulatory effect on the inhibition of aging, as well as the migration and invasion of VSMCs in diseased arteries (6-9). However, little is known about the effects of Buyang Huanwu decoction 
(BYHWD) on aging. BYHWD, a classic TCM formulation featured as Qi-tonifying, stasis-eliminating, and has been used for therapy of stroke for centuries $(10,11)$. Interestingly, cumulative evidence revealed that one of the mechanisms of BYHWD on various diseases represents a target to vascularity $(10,11)$. For example, BYHWD shows a protective effect on cerebral arteries, coronary arteries and pulmonary arteries, etc. (11), the underlying molecular mechanisms of which relies on its pharmacological role in anti-inflammation, antioxidative, anti-apoptosis and anti- angiotensin II (Ang II) (12-14). Of note, it has been shown that BYHWD could inhibit VSMC proliferation caused by injury- or plateletderived growth factor $(15,16)$. In this scenario, there is potential role for BYHWD to control age-associated migration and invasion of serum containing BYHWD (VSMCs).

Age-associated VSMC migration/invasion represents a complex process and is regulated by multiple factors. Ang II is a major inductor of VSMCs senescence, and has been shown to potently simulate VSMCs migration and invasion (17-21). In addition, inhibition of Ang II pathways has been shown to substantially reduce ageassociated arterial remodeling $(3,4)$. The basement membrane which surrounds VSMC is cleaved by matrix metalloprotease (MMP) and restructured for ageassociated migration and invasion (2,3,22). Exposure of new VSMCs to Ang II via activation of MMP- 2 increases the invasive capacity of old cells whereas MMP inhibitor reverses this effect $(23,24)$. Emerging evidence points to sirtuin1 (SIRT1) as a contributer to the regulation of health and lifespan $(25,26)$. It has been demonstrated that over-expression of SIRT1 and an activator of SIRT1 (e.g. resveratrol) markedly inhibits VSMCs migration and invasion $(27,28)$.

Serum pharmacology is generally accepted as a standardized experimental method for use of TCM in in vitro experiments. Serum containing BYHWD (BYHWS) has been developed and described as an in vitro model of BYHWD treatment $(29,30)$. To determine the role of BYHWS in the regulation of age-associated VSMCs migration and invasion, we induced human VSMC senescence by Ang II treatment and cultured VSMC in the presence or absence of BYHWS. We then assessed the consequences of invasion and migration capacities, MMP-2 expression and change of SIRT1 signaling.

\section{Materials and Methods}

\subsection{Animals and preparation of drug-containing serum}

Male Sprague-Dawley (SD) rats, aged 6-8 weeks, were purchased from Guangdong Medical Laboratory Animal Center (Guangzhou, China). All rats were caged under conditions of temperature control with water and food ad libitum. The experimental procedures and protocols were approved by the Institutional Animal Care and Use Committee of Guangdong Pharmaceutical University.

BYHWD was composed of Huangqi (Radix Astragali seu Hedysari), Danggui (Radix Angelica sinensis), Chishao (Radix Paeoniae Rubra), Chuanxiong (Rhizoma Ligustici Chuanxiong), Honghua (Flos Carthami), Taoren (Semen Persicae) and Dilong (Pheretima). The components were purchased from the First Affiliated Hospital of Guangdong Pharmaceutical University (Guangzhou, China) and mixed in a ratio of 120:6:4.5:3:3:3:3 (dry weight). BYHWD was boiled and concentrated to a final concentration of $1 \mathrm{~g} / \mathrm{mL}$ (equivalent to dry weight of raw materials). The $28 \mathrm{SD}$ rats were then randomly divided into 2 groups: control group $(n=12)$ and BYHWD group $(n=16)$. BYHWD (18.5 g/Kg, equivalent to adult human dose) was intragastricly administrated for 7 days (twice per day) according to previous reports $(30,31)$. Control group was treated with distilled water correspondingly. After the rat was fasted for $12 \mathrm{~h}$ and received the last administration of medicine for $90 \mathrm{~min}$, abdominal aortic blood was collected. Blood samples were allowed to clot for $2 \mathrm{~h}$ at $4^{\circ} \mathrm{C}$ and then centrifuged at $1,500 \times \mathrm{g}$ for $20 \mathrm{~min}$. The serum was filtered and stored at $-20^{\circ} \mathrm{C}$ after inactivation at $56^{\circ} \mathrm{C}$ for $30 \mathrm{~min}$. Serum containing BYHWD and control was defined as BYHWS and CS.

\subsection{Cell culture}

Human aorta VSMCs (HA-VSMCs), obtained from ATCC (CRL-1999, Manassas, VA, USA), were cultured in F-12K medium (ATCC) containing 0.05 $\mathrm{mg} / \mathrm{mL}$ ascorbic acid, $0.01 \mathrm{mg} / \mathrm{mL}$ insulin, $0.01 \mathrm{mg} /$ $\mathrm{mL}$ transferrin, $10 \mathrm{ng} / \mathrm{mL}$ sodium selenite, $0.03 \mathrm{mg} / \mathrm{mL}$ endothelial cell growth supplement (ECGS), $10 \mathrm{mM}$ hydroxyethyl piperazine ethanesulfonic acid (HEPES) and $10 \mathrm{mM}$ 2-[Tris(hydroxymethyl)-methylamino]ethanesulfonic acid (TES), and supplemented with $10 \%$ fetal bovine serum (FBS, Gibco, Thermo Fisher Scientific, Inc., Waltham, MA, USA), 10\% BYHWS or $10 \%$ CS. Cells were incubated in humidified $95 \% \mathrm{O}_{2}$ air and $5 \% \mathrm{CO}_{2}$ atmosphere at $37^{\circ} \mathrm{C}$. Medium was renewed every 2 to 3 days. HA-VSMCs were cultured, passaged (less than passage 5) and treated with or without Ang II (100 nM, R\&D research Inc., Minneapolis, MN, USA) for $72 \mathrm{~h}$ to induce senescence.

\subsection{SIRT1 siRNA silence}

HA-VSMCs were transfected with either si-SIRT1 (5 nM, AM 16708) or scrambled siRNA (Silencer Select SiRNA, 40 nM, AM 4635) (Ambion, Carlsbad, CA, USA) using Lipofectamine RNAiMAX Reagent (Invitrogen; Thermo Fisher Scientific, Inc., Waltham, MA, USA) for $6 \mathrm{~h}$ following manufacturer's instructions. The final concentration of si-SIRT1 and scrambled siRNA were $50 \mathrm{nM}$. 


\subsection{Lentiviral activation particles transduction}

HA-VSMCs were seeded at $1 \times 10^{5}$ cells per well in 6-well plates and incubated with complete medium $(10 \%$ FBS) overnight at $37^{\circ} \mathrm{C}$. Transduction was carried out with complete medium containing $3 \mu \mathrm{g} / \mathrm{mL}$ Polybrene (sc-134220, Santa Cruz Biotechnology, Inc., Dallas, TX, USA). Then $10 \mu \mathrm{L}$ of SIRT1 lentiviral activation particles (sc-400085-LAC, Santa Cruz Biotechnology, Inc.) and $10 \mu \mathrm{L}$ of control lentiviral activation particles (sc-437282, Santa Cruz Biotechnology, Inc.) were added to the culture, followed by incubation overnight. The culture medium was then removed and replaced with appropriate treatment.

\subsection{Senescence-associated $\beta$-galactosidase (SA $\beta$-gal) Assay}

HA-VSMCs in 6-well plates were washed twice in PBS, fixed for $5 \mathrm{~min}$ in $4 \%$ paraformaldehyde in PBS, and stained with SA $\beta$-gal (C0602, Beyotime Biotechnology, Shanghai, China) according to the manufacturer's instructions. The percentage of SA $\beta$-gal-expressing cells was examined in 4 randomly selected fields under the microscope (Leica Microsystems GmbH, Wetlzar, Germany).

\subsection{Wound healing migration}

HA-VSMCs were seeded into 24-well plates with inserts in wells (CytoSelect, CBA-120-T, Neobioscience Biotechnology, Shenzhen, China) and grown to confluence. The monolayer cells then generated a $0.9 \mathrm{~mm}$ "wound field" by carefully removing inserts. The cells were allowed to heal for $48 \mathrm{~h}$. Cell stain solution was added to each well for $15 \mathrm{~min}$ and the wounded areas were viewed through a microscope. Quantitative analysis of the cell percent closure was performed using ImageJ software version 1.37 (National Institutes of Health, Bethesda, MA, USA). The level of woundhealing percent closure was evaluated by calculating the percentage of the repopulated cell surface area divided by the cell free area at the initial state. Average data were from at least three independent experiments. Appropriate intervention was performed prior to seeding.

\subsection{Invasion assay}

Serum-induced invasion movement was assessed using modified Boyden chambers equipped with $8 \mu \mathrm{m}$ pore-size polycarbonate filters (PFA8, Neuro probe, Gaithursburg, MD, USA). The upper compartment was coated with BD Matrigel (356234, Solasrbio Science \& Technology Co., Ltd., Shanghai, China) to form a matrix barrier. In total, $2 \times 10^{5}$ cells were suspended in serum-free F-12K complete medium following appropriate treatment and added to the upper chamber. The lower chamber was filled with F-12K complete medium containing 10\% FBS, $10 \%$ BYHWS or $10 \%$ CS as chemoattractant. After a $4 \mathrm{~h}$ incubation period at $37^{\circ} \mathrm{C}$, the cells that had crossed the basement membrane and migrated to the lower side of the filter were fixed and stained with hematoxylin and eosin (Solasrbio Science \& Technology Co., Ltd.). Four random fields were counted at $400 \times$ magnification for each filter.

\subsection{Western blotting analysis}

Western blot was performed according to our previous method (32). Briefly, whole-cell lysates were prepared and quantified. Proteins were then separated by $4-12 \%$ sodium dodecyl sulfate polyacrylamide gel electrophoresis (SDS-PAGE) (Bio-Rad Laboratories, Inc., Hercules, CA, USA) under reducing conditions and transferred onto polyvinylidene difluoride membranes. The transferred membranes were immunoblotted overnight at $4{ }^{\circ} \mathrm{C}$ in phosphate buffer solution (PBS) containing primary antibodies to SIRT1 (04-1557, mouse anti-human monoclonal antibody, 1:1,000, EMD Millipore, Billerica, MA, USA), MMP-2 (AF902, goat anti-human polyclonal antibody, 1:500, R\&D research Inc.), $\beta$-actin (cs-376421, mouse anti-human monoclonal antibody, 1:5,000, Santa Cruz Biotechnology, Inc.) after blocking nonpecific binding. The membranes were then incubated with horseradish peroxidase-conjugated antimouse/goat immunoglobulin G (BA1050 and BA1060, 1:2,000, Wuhan Boster Biological Technology, Ltd., Wuhan, China) at room temperature for $2 \mathrm{~h}$. The density of the visualized bands was quantified using an image analyzer (model GS-700, Bio-Rad Laboratories, Inc.). $\beta$-actin was used as the loading control.

\subsection{Gelatin zymography}

HA-VSMCs were treated and cell supernatant was resolved onto $10 \%$ Novex gelatin zymogram gels (Invitrogen; Thermo Fisher Scientific, Inc.), run at 90 $\mathrm{V}$ for $3 \mathrm{~h}$ at $4^{\circ} \mathrm{C}$. The gels were incubated in Novex zymogram renature buffer (Invitrogen; Thermo Fisher Scientific, Inc.) at room temperature for $30 \mathrm{~min}$ and then transferred to Novex zymogram developing buffer (Invitrogen; Thermo Fisher Scientific, Inc.) at $37^{\circ} \mathrm{C}$ overnight. The gels were photographed after staining with $0.2 \%$ Coomassie blue and quantified using ImageJ (National Institutes of Health).

\subsection{Statistical Analysis}

All results were presented as mean \pm standard error of the mean. All experiments were repeated independently 3 times. Statistical comparisons of multiple groups were made via an ANOVA, followed by Bonferroni post hoc test. The differences between two groups using Student's $t$ tests were two-sided. All statistical analyses 
were performed using GraphPad Prism version 4.00 for windows (GraphPad Software, San Deigo, CA, USA). $P$ $<0.05$ was defined as statistical significance.

\section{Results}

\subsection{BYHWS inhibited the migration and invasion of senescent HA-VSMC}

Previous studies have shown that Ang II is an inductive agent of VSMC premature senescence $(18,19)$. In this study, SA $\beta$-gal activity was measured first in HA-VSMC grown in the presence of Ang II. Cellular premature senescence induced by Ang II is shown in Figure 1A, among which the percentage of SA $\beta$-gal-positive cells was significantly increased $(p<0.05)$. To determine whether BYHWS can affect age-associated migration and invasion of VSMC in vitro, wound-healing and invasion assays were performed in HA-VSMC cultured by treatment with BYHWS. In contrast to control cells, the administration of Ang II significantly promoted the migration of HA-VSMCs to the wounded surface (Figure $1 \mathrm{~B}$ and $1 \mathrm{C}$ ). In addition, the invasion of HA-VSMCs was significantly enhanced in the presence of Ang II
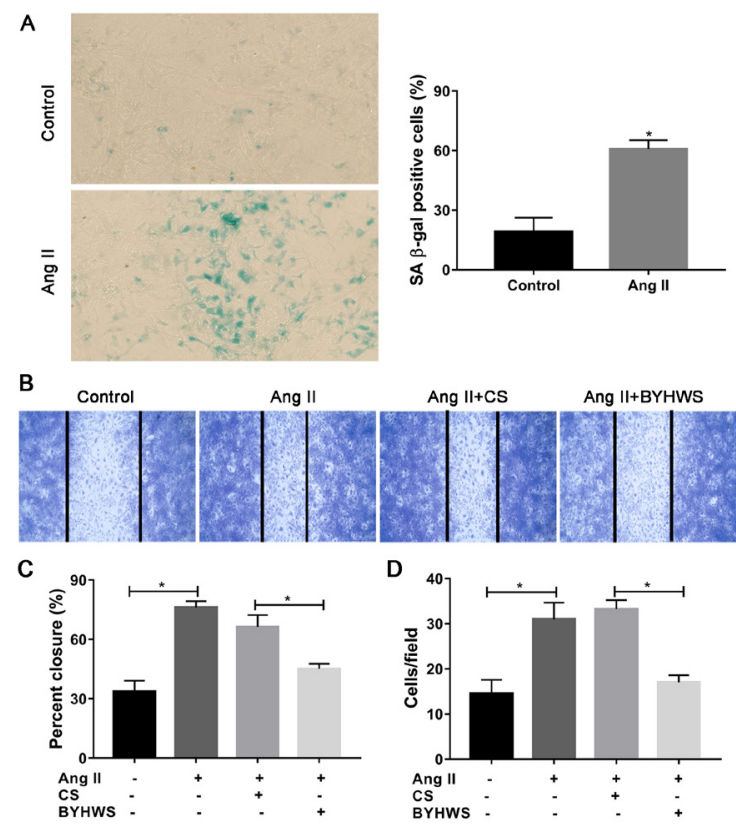

Figure 1. BYHWS inhibited the migration and invasion of senescent HA-VSMC. (A) Representative photomicrographs and average data of SA $\beta$-gal staining in HA-VSMC with and without Ang II treatment. Scale bar indicates $50 \mu \mathrm{m} .{ }^{*} p<0.05$ compared with control. (B) Representative photomicrographs of wound healing assay in HA-VSMC cultured without Ang II or with Ang II treatment in media containing $10 \%$ CS or $10 \%$ BYHWS. Original magnification $\times 40$. (C) Average data of wound healing assay in HA-VSMC cultured without Ang II or with Ang II treatment in media containing $10 \%$ CS or $10 \%$ BYHWS. ${ }^{*} p<0.05$ in comparison between two groups. (D) Average data of invasion analysis of HA-VSMC cultured without Ang II or with Ang II treatment in media containing $10 \% \mathrm{CS}$ or $10 \%$ BYHWS. $* p<0.05$ in comparison between two groups. Ang II, angiotensin II; CS, serum containing vehicle; BYHWS, serum containing Buyang Huanwu decoction. compared to that of the control cells $(p<0.05)$ (Figure 1D). However, when HA-VSMCs in the presence of rat sera were analyzed, we found that the migration and invasion of Ang II-induced senescent cells cultured in $10 \%$ BYHWS were significantly decreased compared to those of cells grown in $10 \%$ CS $(p<0.05)$ (Figure 1B$1 D)$, indicating that BYHWS inhibited migration and invasion of Ang II-induced senescent HA-VSMC.

\subsection{BYHWS reduced MMP-2 production and secretion in senescent HA-VSMC}

Since MMP-2 is a senescence marker and a key regulator in the migration and invasion of VSMCs $(2,3,22,25)$, we conducted Western blotting and gelatin zymography detection to assess the effects of BYHWS on MMP-2 expression. As shown in Figure 2, Ang II treatment led to a significant increase of MMP-2 protein expression and induced its activation. Nevertheless, the treatment with serum containing BYHWD significantly reduced the expression and activation of MMP-2 protein, compared to that treated with medium containing $10 \% \mathrm{CS}(p<0.05)$ (Figure 2A and 2B).

\subsection{BYHWS delayed the down-regulation of SIRT1 protein in senescent HA-VSMCs}

A low SIRT1 level contributed to not only an acceleration of cellular senescence but also increased capacity for cellular migration and invasion $(25-28,33)$. Thus we measured the levels of SIRT1 protein in the Ang II-induced HA-VSMCs. As shown in Figure 3, the amount of SIRT1 protein in HA-VSMCs progressively declined with Ang II treatment. Specifically, a 60\% reduction in SIRT1 protein level was observed in Ang II-induced cells. Of note, in the Ang II-induced model,

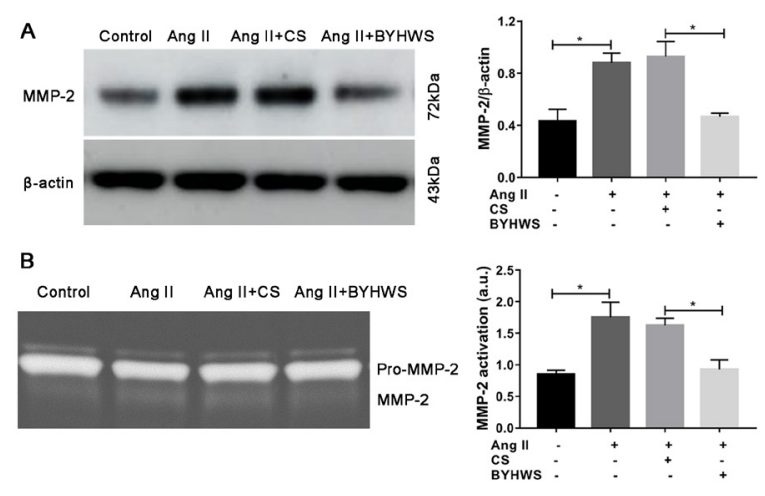

Figure 2. BYHWS reduced MMP-2 expression in senescent HA-VSMC. (A) Representative immunoblots and average data of MMP-2 protein from HA-VSMCs without Ang II or with Ang II treatment in media containing $10 \%$ CS or $10 \%$ BYHWS. $* p<0.05$ in comparison between two groups. (B) Gelatin zymograms of cell supernatant from HA-VSMCs without Ang II or with Ang II treatment in media containing $10 \%$ CS or $10 \%$ BYHWS. ${ }^{*} p<0.05$ in comparison between two groups. Ang II, angiotensin II; CS, serum containing vehicle; BYHWS, serum containing Buyang Huanwu decoction. 
the SIRT1 protein levels in 10\% BYHWS-treated cells were much higher than those found in $10 \%$ CS-treated cells. These results indicated that the administration of BYHWS significantly prevented the down-regulation of

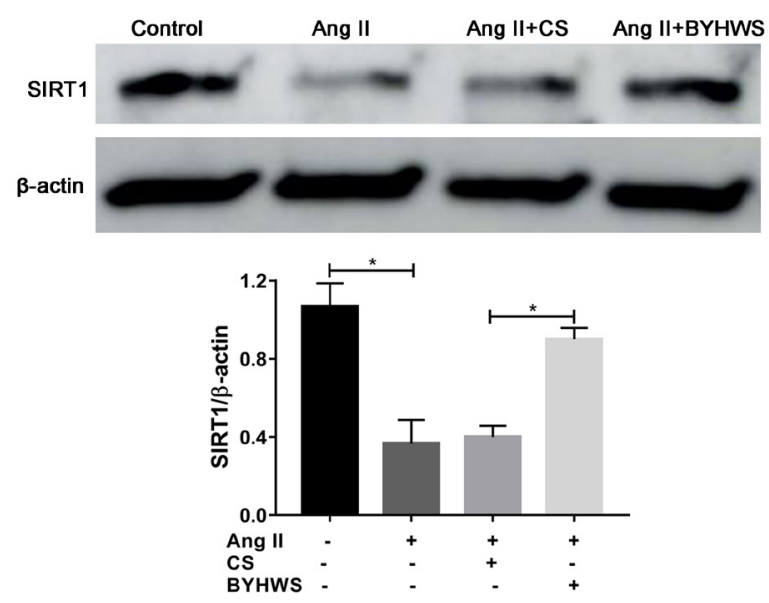

Figure 3. SIRT1 protein decreased in HA-VSMCs with Ang II-induced senescence and BYHWS retarded this effect. Representative immunoblots and average data of SIRT1 protein from HA-VSMCs without Ang II or with Ang II treatment in media containing $10 \%$ CS or $10 \%$ BYHWS. $* p<0.05$ in comparison between two groups. Ang II, angiotensin II; CS, serum containing vehicle; BYHWS, serum containing Buyang Huanwu decoction. age-associated SIRT1 caused by Ang II.

3.4. SIRT1 over-expression decreased the capacity of cell migration and invasion, as well as MMP-2 activation in senescent HA-VSMCs

To investigate the effects of SIRT1 on VSMC migration and invasion, and MMP-2 secretion, SIRT1 overexpression was conducted by transduction, and SIRT1 levels were significantly increased in SIRT1 overexpressed HA-VSMCs compared to negative lentiviral and controls $(p<0.05)$ (Figure 4A). Remarkably, the over expression of SIRT1 in senescent HA-VAMCs significantly reduced migratory and invasive capacity and MMP-2 activation compared to those of control cells $(p<0.05)$ (Figure 4B-4D).

\subsection{SIRT1 was required for BYHWS's effects on the migration and invasion of senescent HA-VSMCs}

We finally investigated the involvement of SIRT1 in the protective effect of BYHWS towards senescent HA-VSMC. Western blot analysis showed that after transfection of HA-VSMCs with si-SIRT1, the expression of SIRT1 protein was statistically down-regulated by approximately $45 \%$ compared to SIRT1 levels in cells

A
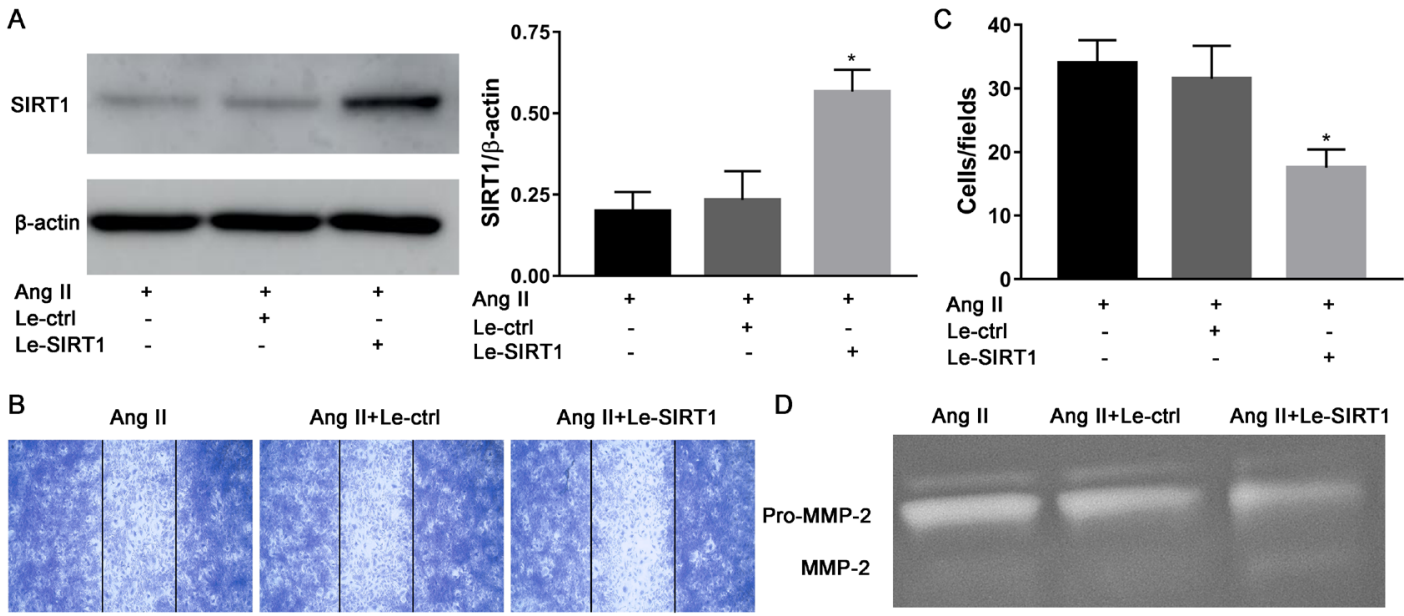

Ang II+Le-SIRT1
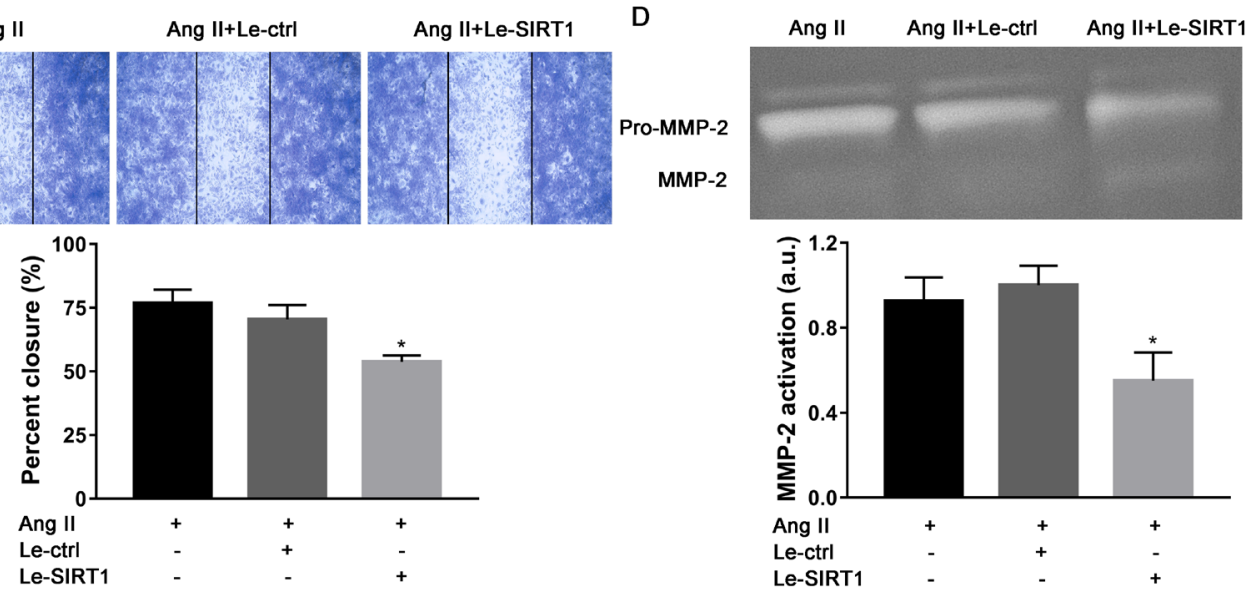

Figure 4. Over-expression of SIRT1 decreased cellular invasion and MMP-2 activation in HA-VSMCs with Ang II-induced senescence. (A) Representative immunoblots and average data of SIRT1 protein in senescent HA-VSMC transduced with Le-SIRT1 or Le-ctrl. ${ }^{*} p<0.05$ compared with Ang II control. (B) Representative average invasion analysis of senescent HA-VSMC transduced with Le-SIRT1 or Le-ctrl. * $p<0.05$ compared with Ang II control. (C) Representative average invasion analysis of HA-VSMC transduced with Le-SIRT1 or Le-ctrl. * $p<0.05$ compared with Ang II control. (D) Gelatin zymograms of cell supernatant from senescent HA-VSMCs transduced with Le-SIRT1 or Le-ctrl. ${ }^{*} p<0.05$ compared with Ang II control group. Ang II, angiotensin II; Le-ctrl, control lentiviral activation particles; Le-SIRT1, SIRT1 lentiviral activation particles. Ang II control group, AngII+, Le-ctrl-, Le-SIRT1-. 
transfected with a negative control siRNA $(p<0.05)$ (Figure 5A). In wound healing and Boyden chamber assays, the decrease of SIRT1 expression dramatically increased the capacity of migration and invasion of senescent HA-VSMCs (Figure 5B and 5C). Moreover, si-SIRT1 also partially counteracted the inhibitory effect of BYHWS on cell migration and invasion (Figure 5B5D). Similarly, transfection of si-SIRT1 in senescent HAVAMCs further significantly increased MMP-2 protein and activation, and statistically abolished the protective effects of BYHWS with inhibition of MMP-2 expression $(p<0.05)$ (Figure 5E and 5F). These data suggested that BYHWS inhibited the migration and invasion of HAVSMCs, via SIRT1 expression in Ang II-treated HAVSMCs.

\section{Discussion}

The study of cellular processes of age-associated migration and invasion in organisms subjected to BYHWD regimens is experimentally challenging. Here, we aimed to determine the effects of BYHWS
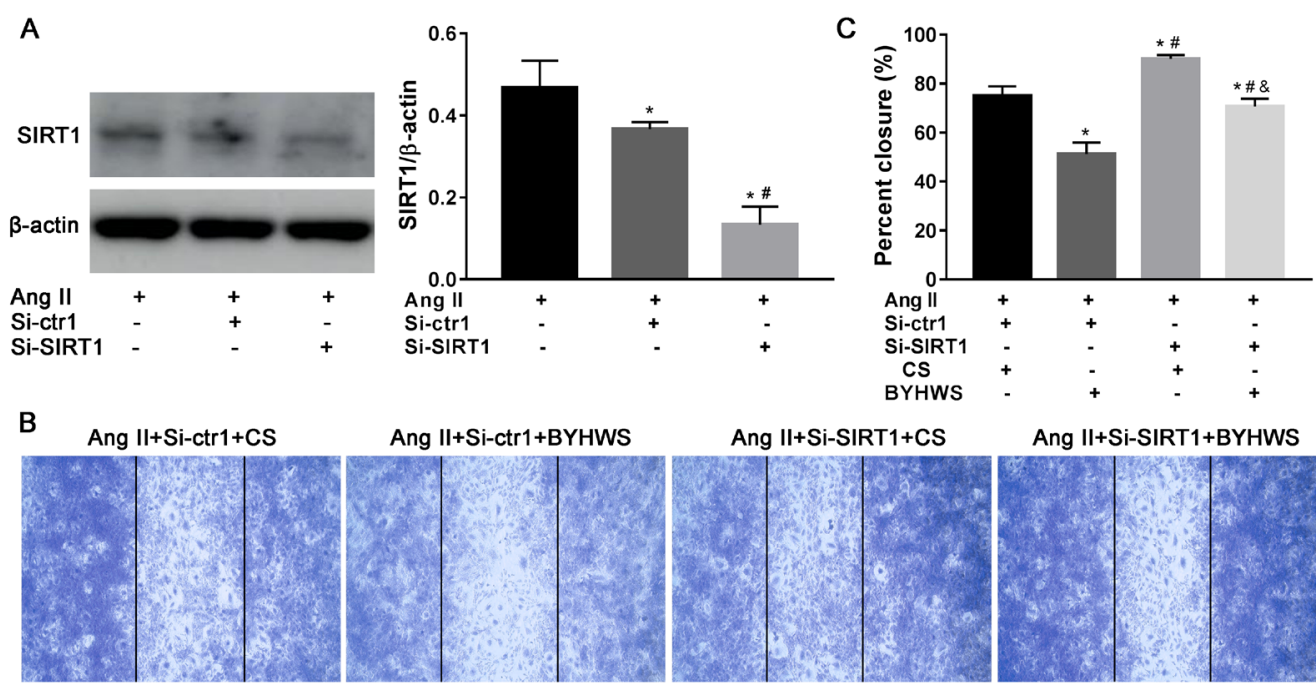

Ang II+Si-ctr1+BYHWS

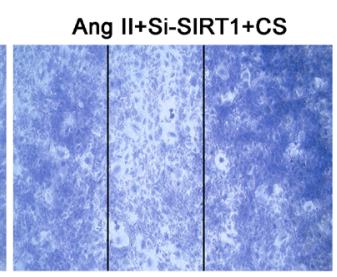

Ang II+Si-SIRT1+BYHWS
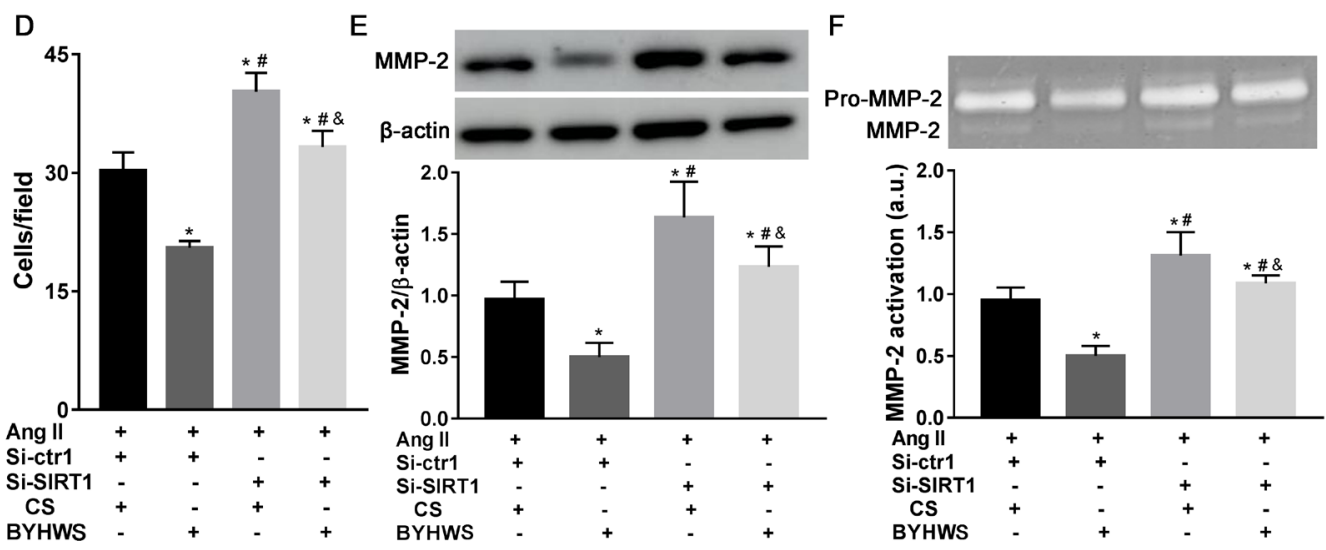

Figure 5. BYHWS modulated HA-VSMCs migration and invasion in a SIRT1-dependent manner. (A) Representative immunoblots and average data of SIRT1 protein in senescent HA-VSMC transfected with si-SIRT1 or si-ctrl. * $p<0.05$ compared with Ang II control group. ${ }^{*} p<0.05$ compared with Si-ctrl group. (B) Representative photomicrographs and average data of wound healing assay in HA-VSMC transfected with si-SIRT1 or si-ctrl, and then treated with Ang II in media containing $10 \%$ CS or $10 \%$ BYHWS . Original magnification $\times 40 .{ }^{*} p<0.05$ compared with $10 \%$ CS control group. ${ }^{*} p<0.05$ compared with $10 \%$ BYHWS group. \& $p<0.05$ compared with $10 \%$ CS + Si-SIRT1 group. (C) Representative average invasion analysis of HAVSMC transfected with si-SIRT1 or si-ctrl, and then treated with Ang II in media containing $10 \%$ CS or $10 \%$ BYHWS. $* p<0.05$ compared with $10 \%$ CS control group. ${ }^{\#} p<0.05$ compared with $10 \%$ BYHWS group. ${ }^{\star} p<0.05$ compared with $10 \%$ CS + SiSIRT1 group. (D) Representative immunoblots and average data of MMP-2 protein from HA-VSMCs transfected with si-SIRT1 or si-ctrl, and then treated with Ang II in media containing $10 \%$ CS or $10 \%$ BYHWS. ${ }^{*} p<0.05$ compared with $10 \%$ CS control group. ${ }^{\#} p<0.05$ compared with 10\% BYHWS group. ${ }^{\star} p<0.05$ compared with $10 \%$ CS + Si-SIRT1 group. (E) Representative immunoblots and average data of MMP-2 protein from HA-VSMCs transfected with si-SIRT1 or si-ctrl, and then treated with Ang II in media containing $10 \%$ CS or $10 \%$ BYHWS. ${ }^{*} p<0.05$ compared with $10 \%$ CS control group. ${ }^{*} p<0.05$ compared with $10 \%$ BYHWS group. ${ }^{\&} p<0.05$ compared with $10 \%$ CS + Si-SIRT1 group. (F) Gelatin zymograms of cell supernatant from HA-VSMCs transfected with si-SIRT1 or si-ctrl, and then treated with Ang II in media containing $10 \%$ CS or $10 \%$ BYHWS. * $p<0.05$ compared with $10 \%$ CS control group. ${ }^{*} p<0.05$ compared with $10 \%$ BYHWS group. \& $p<0.05$ compared with $10 \%$ CS + Si-SIRT1 group. Ang II, angiotensin II; CS, serum containing vehicle; BYHWS, serum containing Buyang Huanwu decoction; si-ctrl, scrambled siRNA silence; si-SIRT1, SIRT1 siRNA silence. Ang II control group, Ang II+, Si-ctrl-, Si-SIRT1-. Si-ctrl group, Ang II+, Si-ctrl+, Si-SIRT1-. 10\% CS control group, AngII+, Si-ctrl+, Si-SIRT1-, 10\% CS+, 10\% BYHWS-. 10\% BYHWS group, AngII+, Si-ctrl+, Si-SIRT1-, 10\% CS-, 10\% BYHWS+. 10\% CS + si SIRT1 group, AngII+, Si-ctrl-, Si-SIRT1+, 10\% CS+, 10\% BYHWS-. 
on this cellular process in vitro using Ang II-induced senescent HA-VSMCs. Our results clearly showed that BYHWS could significantly delay age-associated increased migration and invasion of these cells, which was accompanied with reduced MMP-2 expression. In addition, we also identified SIRT1 as a pivotal factor in modulating BYHWS's effects on age-associated HAVSMC responses.

Arterial aging is a cornerstone of systemic aging $(2,3,17)$. The increase of intima thickness is nearly linearly associated with age mainly due to the migration and invasion of VSMCs from the arterial media to the intimal $(2,3,34)$. While cumulative studies demonstrated that the capacity of migration and invasion was decreased in senescent VSMCs $(35,36)$. Here, we used a model of HA-VSMC senescence induced by Ang II and verified that senescent HA-VSMCs enhanced migration and invasion, which was in agreement with previous reports regarding increasing migratory and invasive capacities in senescent rat VSMCs $(24,37)$. Furthermore, it has been shown that Ang II can stimulate VSMC migration and invasion measured by a wound healing approach and Boyden chamber assay $(20,21)$.

The precise roles mediating aging and age-associated events related with BYHWD are not fully understood. TCM of Qi-tonifying and stasis- eliminating may play a role in delaying aging, mainly due to the well-known theory that "Qi deficiency and blood stasis" is essential for human aging (38). In terms of vascular aging, it has been proved that TCM based on invigorating Qi and activating blood had effects on delaying VSMC senescence and lowering age-associated increase of VSMC proliferation (6). As a classic TCM formulation of invigorating Qi and activating blood, BYHWD can be used to treat many disorders with Qi deficiency and blood stasis, which is likely to target the specific pathogenesis of vascular diseases (11). BYHWD was previously shown to inhibit aortic intimal hyperplasia and cellular proliferation in cultured $\operatorname{VSMC}(15,16)$. In the current study we demonstrated for the first time that BYHWS treatment inhibited the migration and invasion of senescent HA-VSMCs. However, similar inhibition was not observed in CS-treated senescent HAVSMCs. Other beneficial effects of BYHWD appear to promote the migration of neural precursor cells to ischemic brain areas, thus facilitating its neuroprotective effect (39). Under this condition, cellular migration is a nonpathogenic process for tissue repair in response to ischemic injury, which is different than the age-increased pathological process.

Uncontrolled MMPs, potently activated by Ang II, can cleave both cellular basement membrane and elastin fibers around VSMCs, which results in degradation of extracellular matrix, enabling VSMCs to migration, invasion and proliferation with advanced aging. In cultured VSMCs, both MMP-2 and MMP-9 are related to the migration and invasion of VSMCs $(2,3,22)$. It has been shown that MMP-9 is less important than MMP-2 in triggering intimal thickening in coronary artery rings ex vivo (40). Moreover, MMP-2 expression becomes increased within aging arterial walls, particularly in the thickened intima of different species including humans $(2,3,22,23)$. Here, we showed that the administration of Ang II in HA-VSMCs enhanced MMP-2 expression whereas BYHWS inhibited this promoting effect. These results indicate that BYHWS treatment might retard ageassociated migration and invasion through inhibition of MMP-2 in HA-VSMCs.

SIRT1 (member of the sirtuin family) is a nicotinamide adenosine dinucleotide (NAD)-dependent deacetylase that removes acetyl groups from various proteins. It has been implicated that Sirt1 can impact a wide array of proteins, such as peroxisome proliferatoractivated receptor-gamma and its coactivator-1alpha, forkhead transcriptional factors, AMP-activated protein kinase, NF-kappaB and protein tyrosine phosphatase involved in cardiovascular and metabolic diseases, through regulating metabolic and physiologic processes including stress resistance, metabolism, apoptosis and energy balance (41-43). Notably, in the cardiovascular system, activation of SIRT1 can not only protect against oxidative stress at the cellular level, but can also elevate survival at the systemic level to reduce the risk of coronary heart disease and cerebrovascular disease (42). In the present study, SIRT1 levels in vitro as HA-VSMCs were decreased and senescence was induced. This is consistent with previous studies concerning the reduction of SIRT1 levels in senescent human VSMCs, endothelial cells and fibroblasts $(25,44,45)$. Interestingly, when these senescent HA-VSMCs were cultured in the presence of BYHWS, much higher SIRT1 was induced than the same cells cultured in CS. Therefore, BYHWS protects cells against the decrease of SIRT1 associated with the senescence process. Although a study has reported that BYHWS is involved in a neuron protective role through suppression of the P53 pathway (46), to our knowledge, no previous studies have investigated whether BYHWS directly influences SIRT1 expression. It has been demonstrated that SIRT1's predominate function is in delaying aging and suppressing arterial remodeling $(26,27,33)$. Our further experiments revealed that down regulation of SIRT1 levels by siRNA-knockdown elevated cellular migration/invasion and MMP-2, leading to enhancement in senescent HA-VSMCs. Of note, the suppression of SIRT1 reversed BYHWS-mediated inhibition of MMP-2 levels and the migration/invasion in senescent HA-VSMCs. Conversely, over-expression of SIRT1 in senescent HA-VSMCs resulted in reduced expression of MMP2 and was also consistent with concomitant decreases in cellular migration and invasion. Cumulative evidence unraveled that SIRT1 plays a pivotal role in the treatment of cardiovascular disease, through which, hydroxytyrosol, mitochondrial aldehyde dehydrogenase (ALDH2), and sulforaphane (SFN) 
showed great promise for providing protection (47-50). Our data suggest that BYHWS regulation of cellular migration/invasion and MMP-2 expression in HAVSMCs is dependent on activation of SIRT1. However, in vitro experiments are still required to investigate the effect of BYHWS in clinical practice, and the therapeutic value of combined use of BYHWS, ALDH2, SFN, etc. for instance, and requires further evaluation.

\section{Conclusion}

This study demonstrated that treatment with BYHWS in senescent HA-VSMCs resulted in $i$ ) reduced capacity of migration and invasion, $i$ ) decreased MMP-2 expression, and iii) sustained the increase of SIRT1 levels. Our data also showed that modulation of SIRT1 levels by either knockdown or over-expression of this protein promoted or inhibited age-associated VSMC migration and invasion. Moreover, BYHWS has an effect on ageassociated VSMC responses in a SIRT1-dependent manner.

\section{Acknowledgements}

This work was support by the National Natural Science Foundation of China (No. 81774241) and Science and Technology Program of Shenzhen (No. JCYJ201504011163247220).

\section{References}

1. Harvey A, Montezano AC, Touyz RM. Vascular biology of ageing-Implications in hypertension. J Mol Cell Cardiol. 2015; 83:112-121.

2. Wang M, Jiang L, Monticone RE, Lakatta EG. Proinflammation: The key to arterial aging. Trends Endocrinol Metab. 2014; 25:72-79.

3. Zhang G, Liu Z, Cui G, Wang X, Yang Z. MicroRNA486-5p targeting PIM-1 suppresses cell proliferation in breast cancer cells. Tumor Biology. 2014; 35:11137.

4. Lakatta EG. So! What's aging? Is cardiovascular aging a disease? J Mol Cell Cardiol. 2015; 83:1-13.

5. Nilsson PM. Early vascular aging (EVA): Consequences and prevention. Vascular health and risk management. 2008; 4:547-552.

6. Tao LL, Lei Y, Wang GL, Zhu LQ, Wang Y. Effect of extracts from Radix Ginseng, Radix Notoginseng and Rhizoma Chuanxiong on delaying aging of vascular smooth muscle cells in aged rats. Chin J Integr Med. 2012; 18:582-590.

7. Ge X, Chen S, Liu M, Liang T, Liu C. Evodiamine Attenuates PDGF-BB-Induced Migration of Rat Vascular Smooth Muscle Cells through Activating PPARgamma. Int J Mol Sci. 2015; 16:28180-28193.

8. Hwang SM, Lee YJ, Lee YP, Yoon JJ, Lee SM, Cha JD, Choi KM, Kang DG, Lee HS. Anti-Proliferative Effect of an Aqueous Extract of Prunella vulgaris in Vascular Smooth Muscle Cells. Evid Based Complement Alternat Med. 2013; 2013:936463.

9. Shih HC, Chang KH, Chen FL, Chen CM, Chen SC, Lin YT, Shibuya A. Anti-aging effects of the traditional
Chinese medicine bu-zhong-yi-qi-tang in mice. Am J Chin Med. 2000; 28:77-86.

10. Zhang W, Gao K, Liu J, Zhao H, Wang J, Li Y, Murtaza G, Chen J, Wang W. A review of the pharmacological mechanism of traditional Chinese medicine in the intervention of coronary heart disease and stroke. Afr J Tradit Complement Altern Med. 2013; 10:532-537.

11. Li JH, Liu AJ, Li HQ, Wang Y, Shang HC, Zheng GQ. Buyang huanwu decoction for healthcare: Evidence-based theoretical interpretations of treating different diseases with the same method and target of vascularity. Evid Based Complement Alternat Med. 2014; 2014:506783.

12. Jin Y, Dong L, Wu C, Qin J, Li S, Wang C, Shao X, Huang D. Buyang Huanwu Decoction fraction protects against cerebral ischemia/reperfusion injury by attenuating the inflammatory response and cellular apoptosis. Neural Regen Res. 2013; 8:197-207.

13. Liu Y, Lin R, Shi X, Fang Z, Wang W, Lin Q, Zhang J, Zhang H, Ji Q. The roles of buyang huanwu decoction in anti-inflammation, antioxidation and regulation of lipid metabolism in rats with myocardial ischemia. Evid Based Complement Alternat Med. 2011; 2011:561396.

14. Ren J, Lin C, Liu J, Xu L, Wang M. Experimental study on Qi deficiency and blood stasis induced by muti-factor stimulation in rats. Zhongguo Zhong Yao Za Zhi. 2011; 36:72-76. (in Chinese)

15. Chen G, Wu L, Deng CQ. The effects of BuYang HuanWu Decoction and its effective components on proliferationrelated factors and ERK1/2 signal transduction pathway in cultured vascular smooth muscle cells. J Ethnopharmacol. 2011; 135:7-14.

16. Wu L, Zhang W, Li H, Chen BY, Zhang GM, Tang YH, He FY, Deng CQ. Inhibition of aortic intimal hyperplasia and cell cycle protein and extracellular matrix protein expressions by BuYang HuanWu Decoction. J Ethnopharmacol. 2009; 125:423-435.

17. Wang M, Khazan B, Lakatta EG. Central Arterial Aging and Angiotensin II Signaling. Curr Hypertens Rev. 2010; 6:266-281.

18. Kunieda T, Minamino T, Nishi J, Tateno K, Oyama T, Katsuno T, Miyauchi H, Orimo M, Okada S, Takamura M, Nagai T, Kaneko S, Komuro I. Angiotensin II induces premature senescence of vascular smooth muscle cells and accelerates the development of atherosclerosis via a p21dependent pathway. Circulation. 2006; 114:953-960.

19. Zhao L, Li AQ, Zhou TF, Zhang MQ, Qin XM. Exendin-4 alleviates angiotensin II-induced senescence in vascular smooth muscle cells by inhibiting Racl activation via a cAMP/PKA-dependent pathway. Am J Physiol Cell Physiol. 2014; 307:C1130-1141.

20. Li Y, Wang N, Chen C, He D, Yang J, Zeng C. Inhibitory effect of D3 dopamine receptor on migration of vascular smooth muscle cells induced by synergistic effect of angiotensin II and aldosterone. Clin Exp Hypertens. 2015; 37:288-293.

21. Pantan R, Tocharus J, Phatsara M, Suksamrarn A, Tocharus C. Synergistic effect of atorvastatin and cyanidin-3-glucoside against angiotensin II-mediated vascular smooth muscle cell proliferation and migration through MAPK and PI3K/Akt pathways. Arch Pharm Res. 2016.

22. Wang M, Kim SH, Monticone RE, Lakatta EG. Matrix metalloproteinases promote arterial remodeling in aging, hypertension, and atherosclerosis. Hypertension. 2015; 65:698-703. 
23. Wang M, Zhang J, Spinetti G, Jiang LQ, Monticone R, Zhao D, Cheng L, Krawczyk M, Talan M, Pintus G, Lakatta EG. Angiotensin II activates matrix metalloproteinase type II and mimics age-associated carotid arterial remodeling in young rats. Am J Pathol. 2005; 167:1429-1442.

24. $\mathrm{Fu} \mathrm{Z}$, Wang $\mathrm{M}$, Gucek $\mathrm{M}$, et al. Milk fat globule protein epidermal growth factor-8: A pivotal relay element within the angiotensin II and monocyte chemoattractant protein-1 signaling cascade mediating vascular smooth muscle cells invasion. Circ Res. 2009; 104:1337-1346.

25. de Cabo R, Liu L, Ali A, Price N, Zhang J, Wang M, Lakatta E, Irusta PM. Serum from calorie-restricted animals delays senescence and extends the lifespan of normal human fibroblasts in vitro. Aging (Albany NY). 2015; 7:152-166.

26. Baur JA, Ungvari Z, Minor RK, Le Couteur DG, de Cabo $\mathrm{R}$. Are sirtuins viable targets for improving healthspan and lifespan? Nat Rev Drug Discov. 2012; 11:443-461.

27. Li L, Zhang HN, Chen HZ, et al. SIRT1 acts as a modulator of neointima formation following vascular injury in mice. Circ Res. 2011; 108:1180-1189.

28. Lin YC, Chen LH, Varadharajan T, Tsai MJ, Chia YC, Yuan TC, Sung PJ, Weng CF. Resveratrol inhibits glucoseinduced migration of vascular smooth muscle cells mediated by focal adhesion kinase. Molecular nutrition \& food research. 2014; 58:1389-1401.

29. Lou L, Zhou J, Liu Y, Wei YI, Zhao J, Deng J, Dong B, Zhu L, Wu A, Yang Y, Chai L. Chlorogenic acid induces apoptosis to inhibit inflammatory proliferation of IL-6induced fibroblast-like synoviocytes through modulating the activation of JAK/STAT and NF-kappaB signaling pathways. Experimental and therapeutic medicine. 2016; 11:2054-2060.

30. Yu P, Guan L, Zhou L, Guo J, Guo R, Lin R, Ding W, Li X, Liu W. Upregulation of glutamate metabolism by BYHWD in cultured astrocytes following oxygenglucose deprivation/reoxygenation in part depends on the activation of p38 MAPK. Exp Ther Med. 2017; 13:30893096.

31. Zhou YC, Liu B, Li YJ, Jing LL, Wen G, Tang J, Xu X, Lv ZP, Sun XG. Effects of buyang huanwu decoction on ventricular remodeling and differential protein profile in a rat model of myocardial infarction. Evidence-based complementary and alternative medicine : eCAM. 2012; 2012:385247.

32. Zhang L, Wu SZ, Ruan YJ, Hong L, Xing XW, Lai WY. Testosterone therapy delays cardiomyocyte aging via an androgen receptor-independent pathway. Braz J Med Biol Res. 2011; 44:1118-1124.

33. Zhang MJ, Zhou Y, Chen L, Wang X, Pi Y, Long CY, Sun MJ, Chen X, Gao CY, Li JC, Zhang LL. Impaired SIRT1 promotes the migration of vascular smooth muscle cellderived foam cells. Histochem Cell Biol. 2016; 146:33-43.

34. Schmidt-Trucksass A, Grathwohl D, Schmid A, Boragk R, Upmeier C, Keul J, Huonker M. Structural, functional, and hemodynamic changes of the common carotid artery with age in male subjects. Arterioscler Thromb Vasc Biol. 1999; 19:1091-1097.

35. Thompson AM, Wagner R, Rzucidlo EM. Age-related loss of SirT1 expression results in dysregulated human vascular smooth muscle cell function. Am J Physiol Heart Circ Physiol. 2014; 307:H533-541.

36. Zhao W, Zheng XL, Peng DQ, Zhao SP. Myocyte enhancer factor $2 \mathrm{~A}$ regulates hydrogen peroxide- induced senescence of vascular smooth muscle cells via microRNA-143. J Cell Physiol. 2015; 230:2202-2211.

37. Wang M, Spinetti G, Monticone RE, Zhang J, Wu J, Jiang L, Khazan B, Telljohann R, Lakatta EG. A local proinflammatory signalling loop facilitates adverse ageassociated arterial remodeling. PLoS One. 2011; 6:e16653.

38. Liu LY, Sun ZQ, Xiang H. Studies on neuro-immunologic regulation of senile rats by using the principle of replenishing qi and promoting blood circulation. Zhongguo Zhong Xi Yi Jie He Za Zhi. 1997; 17:616-619. (in Chinese)

39. Kong X, Su X, Zhu J, Wang J, Wan H, Zhong M, Li L, Lin $\mathrm{N}$. Neuroprotective effect of buyang huanwu decoction on rat ischemic/reperfusion brain damage by promoting migration of neural precursor cells. Rejuvenation Res. 2014; 17:264-275.

40. Louis SF, Zahradka P. Vascular smooth muscle cell motility: From migration to invasion. Exp Clin Cardiol. 2010; 15:e75-85.

41. Pillarisetti S. A review of Sirt1 and Sirt1 modulators in cardiovascular and metabolic diseases. Recent patents on cardiovascular drug discovery. 2008; 3:156-164.

42. Chong ZZ, Wang S, Shang YC, Maiese K. Targeting cardiovascular disease with novel SIRT1 pathways. Future cardiology. 2012; 8:89-100.

43. Ma L, Li Y. SIRT1: Role in cardiovascular biology. Clinica chimica acta; international journal of clinical chemistry. 2015; 440:8-15.

44. Gorenne I, Kumar S, Gray K, Figg N, Yu H, Mercer J, Bennett M. Vascular smooth muscle cell sirtuin 1 protects against DNA damage and inhibits atherosclerosis. Circulation. 2013; 127:386-396.

45. Ota H, Akishita M, Eto M, Iijima K, Kaneki M, Ouchi Y. Sirt1 modulates premature senescence-like phenotype in human endothelial cells. J Mol Cell Cardiol. 2007; 43:571-579.

46. Qu HD, Tong L, Shen JG. Effect of buyang huanwu decoction drug serum on expression of p53 and p 21 genes in cultured rat's cerebral cortical neuron after hypoxia in vitro. Zhongguo Zhong Xi Yi Jie He Za Zhi. 2004; 24:133-135. (in Chinese)

47. Wang W, Jing T, Yang X, He Y, Wang B, Xiao Y, Shang C, Zhang J, Lin R. Hydroxytyrosol regulates the autophagy of vascular adventitial fibroblasts through the SIRT1-mediated signaling pathway. Canadian journal of physiology and pharmacology. 2018; 96:88-96.

48. Wang S, Wang C, Turdi S, Richmond KL, Zhang Y, Ren J. ALDH2 protects against high fat diet-induced obesity cardiomyopathy and defective autophagy: Role of CaM kinase II, histone H3K9 methyltransferase SUV39H, Sirt1, and PGC-1alpha deacetylation. International journal of obesity. 2018

49. Han D, Wang J, Ma S, Chen Y, Cao F. SIRT1 as a Promising Novel Therapeutic Target for Myocardial Ischemia Reperfusion Injury and Cardiometabolic Disease. Current drug targets. 2017; 18:1746-1753.

50. Li YP, Wang SL, Liu B, Tang L, Kuang RR, Wang XB, Zhao C, Song XD, Cao XM, Wu X, Yang PZ, Wang LZ, Chen AH. Sulforaphane prevents rat cardiomyocytes from hypoxia/reoxygenation injury in vitro via activating SIRT1 and subsequently inhibiting ER stress. Acta pharmacologica Sinica. 2016; 37:344-353.

(Received April 13, 2018; Revised May 30, 2018; Accepted June 10, 2018) 